\title{
Serotonin Synthesis and Distribution in Drosophila Dopa Decarboxylase Genetic Mosaics
}

\author{
Ana María Vallés ${ }^{a}$ and Kalpana White \\ Department of Biology, Brandeis University, Waltham, Massachusetts 02254
}

In Drosophila, the enzyme dopa decarboxylase (DDC) catalyzes the last step in the biosynthesis of serotonin (5HT) and dopamine. In the present study, the distribution of DDC and one of its biosynthetic products (5HT) was determined in CNSs that were genetic mosaics, composed of neurons that have DDC activity and neurons that lack DDC activity. Phenotypic mosaicism, that is, immunoreactive (IR) and nonIR neurons within the same nervous system, was observed for both DDC and 5HT immunoreactivity. DDC-IR neurons were always 5HT-IR, but some 5HT-IR neurons devoid of DDC immunoreactivity were also observed. 5HT-IR neurons devoid of DDC immunoreactivity were always in close apposition to other DDC-IR neurons. We suggest that in vivo uptake mechanisms are responsible for $5 \mathrm{HT}$ accumulation in neurons devoid of DDC immunoreactivity.

In Drosophila, a vital locus, dopa decarboxylase ( $D d c)$, encodes the enzyme dopa decarboxylase (DDC) (Wright et al., 1976). The $D d c$-encoded enzyme activity is necessary for catecholamine biosynthesis in the epidermis and the CNS (reviewed in Wright, 1987). It is also essential for the synthesis of serotonin (5HT) in the CNS (Livingstone and Tempel, 1983). Antibodies against 5HT and DDC have been uscd to identify 5HT-immunoreactive (5HT-IR) (Vallés and White, 1988) and DDCimmunoreactive (DDC-IR) neuronal subsets in the Drosophila CNS (Beall and Hirsh, 1987; Konrad and Marsh, 1987). As expected, 5HT-IR neurons are always DDC-IR; in addition, catecholamine-containing neurons are also DDC-IR (Beall and Hirsh, 1987; Konrad and Marsh, 1987).

Certain genetic variants at the $D d c$ locus completely eliminate the gene product. Thus, it is possible to generate animals that are devoid of DDC activity; in these $D d c^{-}$animals, 5HT immunoreactivity is not detected (Vallés and White, 1986a). We have taken advantage of these genetic variants to study the pattern and development of 5HT neurons in the absence of 5HT synthesis (Vallés and White, 1986a; Budnik et al., 1989). This pattern in mutant CNSs, which are devoid of endogenous 5HT, can be revealed by incubating the CNSs in 5HT, because monoamine neurons can specifically accumulate exogenously supplied

Received Feb. 22, 1990; revised June 26, 1990; accepted July 6, 1990.

We thank Dr. Jay Hirsh for the generous gift of DDC antibody and Dr. Don Gailey for the $D d c^{+} \cdot \operatorname{Ring} X$ chromosome. We appreciate comments on the manuscript by Dr. Wendi Neckameyer and Dr. Jeff Hall. Parts of this work were submitted by A.M.V. in partial fulfillment of the requirements for the degree of Doctor of Philosophy at Brandeis University. This research was supported by NIH Grant NS 23510 to K.W.

Correspondence should be addressed to Kalpana White at the above address.

"Present address: Ecole Normale Supérieure, Laboratoire de Physiopathologie du Dévelopment, 46 Rue d'Ulm, 75230 Paris Cedex 05, France.

Copyright (c) 1990 Society for Neuroscience $0270-6474 / 90 / 113646-07 \$ 03.00 / 0$ amine. In the mutant, the 5HT neuronal pattern, as revealed by specific uptake, resembles the normal pattern (Vallés and White, 1986a). Therefore, in $D d c^{-}$mutants, neurons committed to the 5HT differentiation pathway (5HT neurons) survive and are able to develop properties required for the specific uptake system as judged by their selective uptake ability, even in the absence of endogenous 5IT synthesis.

Recently, it has been possible to generate $D d c$ genetic mosaics, that is, flies made up of cells that are of 2 different genotypes, $D d c^{-}$and $D d c^{+}$(Gailey et al., 1987). Analyses of adult $D d c$ mosaics revealed that, while there was no absolute requirement for the wild-type $D d c$ function either in the nervous system or in the epidermis, very large mutant clones were deleterious, because they affected the viability of the fly (Gailey et al., 1987). A CNS from a $D d c$ mosaic can potentially be composed of 2 classes of cells: neurons that are $D d c^{+}$, and thus capable of 5HT synthesis, and neurons that do not carry the wild-type allele, and thus are unable to synthesize 5HT. Such mosaic CNSs provide a unique situation where in vivo distribution of 5HT synthesis is modified such that neurons capable of 5HT synthesis, that is, those that are genetically $D d c^{+}$, are only a subset of the 5HT neuronal set. In the present paper, we report an analysis of $D d c$ mosaic CNSs, where we compare 5HT distribution and synthesis. 5HT distribution was assessed by 5HT immunoreactivity, and 5HT synthesis by DDC immunoreactivity. Specifically, we were interested in determining whether within the same CNS, 5HT-IR and 5HT-minus patches could be observed. We also examined whether, in $D d c$ mosaics, all 5HT-IR neurons show DiC immunoreactivity or whether some 5HT-IR neurons lack DDC immunoreactivity. The latter situation could presumably arise if $D d c^{-}$neurons incapable of endogenous 5HT synthesis can accumulate it through in vivo uptake of $5 \mathrm{HT}$ available from $D d c^{+}$neurons within the same CNS.

Part of this work was presented as an abstract (Vallés and White, 1986b).

\section{Materials and Methods}

Genetic stocks. The generation and use of $D d c^{\dagger} \cdot \operatorname{Ring} X$ chromosome, an unstable chromosome carrying the $D d c^{+}$allele, has been previously described by Gailey et al. (1987), who also described all other genetic markers and chromosomes used in this study. The $D d c^{n 27}$ allele is a small deletion in the $D d c$ gene (Gilbert et al., 1984). All broods were raised at $25^{\circ} \mathrm{C}$ in vials containing standard media of molasses, agar, yeast, and cornmeal.

Generation of mosaics. To generate genetic mosaics, broods with $D d c^{+}$. RingX/Binsn; $D d c^{n 27 / S M 6}$ females and cho $s n / Y$; $D d c^{n 27} / S M 6$ males were established. One sixteenth of the progeny, $D d c^{+} \cdot R i n g X /$ cho $s n$; $D d c^{n 27} / D d c^{n 27}$ female zygotes, have a potential to lose the $D d c^{+} \cdot \operatorname{Ring} X$ chromosome and result in a $D d c$ gynandromorph. In the $D d c$ genctic mosaic, the diplo- $X$ cellular patches will be of $D d c^{+} \cdot \operatorname{Ring} X /$ cho sn; 
$D d c^{127} / D d c^{127}$ genotype and wild-type in phenotype, whereas the haplo- $X$ cellular patches will be of $c h o s n ; D d c^{n 27} / D d c^{n 27}$ genotype and thus $D d c^{-}$ and reveal the $X$-linked recessive markers. Male tissues in mosaic larvae were identified by chocolate malpighian tubules, the presence of male gonads, and reduced pigment in the mouth hooks and posterior spiracles. The reduction in pigment is due to lack of sclerotization in the absence of $D D C$. The broods to generate mosaics were transferred every day to fresh vials. Five to $6 \mathrm{~d}$ after egg deposition, when third instar larvae started to wander, larvae were separated from food and inspected in a dissecting microscope for gynandromorphic larvae.

5HT immunocytochemistry. Details of 5HT immunocytochemistry have been previously described (Vallés and White, 1986a). In brief, the CNSs were dissected in ice-cold calcium-free Drosophila Ringer's solution ( $\mathrm{NaCl}, 130 \mathrm{~mm} ; \mathrm{KCl}, 4.7 \mathrm{~mm} ; \mathrm{MgCl}_{2}, 1.8 \mathrm{~mm} ; \mathrm{KH}_{2} \mathrm{PO}_{4}, 0.74$ $\mathrm{mm} ; \mathrm{Na}_{2} \mathrm{HPO}_{4}, 0.35 \mathrm{~mm}$ ). The dissected CNSs were fixed in $4 \%$ paraformaldehyde in $0.1 \mathrm{~m}$ phosphate buffer $(\mathrm{pH}, 7.2)$, washed, then incubated in a 1:200 dilution of primary antibody (monoclonal supernatant from Accurate Chemical and Scientific Corp., MASO55, clone YC5/ 45). After the primary incubation, the CNSs were washed and incubated in goat anti-rat IgG conjugated to fluorescein isothiocyanate (FITC). Washed C.NSs were given a final rinse in $4 \mathrm{~mm}$ sodium carbonate buffer $(\mathrm{pH}, 9.5)$ and mounted in $80 \%$ glycerol in $20 \mathrm{mM}$ carbonate buffer containing $n$-propyl gallate.

$5 H T$ and DDC immunocytochemistry. Labeling CNSs with both 5HT and DDC antibodies was performed essentially as in Beall and Hirsh (1987), with some modifications. CNSs were dissected in calcium-free Drosophila Ringer's solution, then fixed in 4\% paraformaldehyde (Polyscience) buffered in PBS $\left(\mathrm{Na}_{2} \mathrm{PH}_{4}, 7 \mathrm{~mm} ; \mathrm{NaH}_{2} \mathrm{PO}_{4}, 3 \mathrm{~mm} ; \mathrm{NaCl}_{2}, 130\right.$ $\mathrm{mM}$ ). Subsequent washes were performed in the PBS containing $0.1 \%$ BSA and $0.1 \%$ Triton X-100. Samples were incubated first with antiDDC rabbit antibody, followed by a 5 -hr incubation with the rat anti$5 \mathrm{HT}$. After washes, both secondary antibodies were added simultaneously and reacted for $4 \mathrm{hr}$. The secondary antibodies were goat FITCconjugated anti-rat IgG and goat rhodamine-conjugated anti-rabbit IgG used at $1 / 20$ dilution. For details on specificity of the $5 \mathrm{HT}$ monoclonal antibody, see Vallés and White (1986a); for the DDC antiserum, see Beall and Hirsh (1987).

Analyses of immunoreactivity patterns. Mounted samples were visualized in a Zeiss epifluorescent microscope equipped with filters appropriate for FITC and rhodamine fluorescence. Each whole-mount was scored for the presence or absence of each of the 14 ventral ganglion (3 subesophageal, 3 thoracic, and 8 abdominal) 5HT clusters. Additionally, the degree of immunoreactivity in each cluster and in the neuropil of each neuromere was noted. One of 3 categories, normal, low, or absent, was assigned for each parameter.

\section{Results and Discussion}

For the present study, we chose to analyze CNSs of mosaic animals in the larval stage for several reasons: (1) The pattern of $5 \mathrm{HT}$ immunoreactivity in the larval CNS in whole-mount preparations has been previously described (Vallés and White, 1986a, 1988). (2) Within the ventral ganglion, this pattern is relatively simple and robust, and the presence or absence of IR neuronal cell bodies, axons, and dendritic arbors can be reliably scored (see Fig. 1 $A$ ). (3) Larval CNS dissections are easier than adult CNS dissections, and immunostaining works more reliably. (4) The pattern of DDC immunoreactivity in larval CNS whole-mount preparations has also been described (Beall and Hirsh, 1987; Konrad and Marsh, 1987). Although the 5HT-IR ncurons are a subset of DDC-IR neurons, as catecholaminecontaining neurons are also DDC-IR, the 5HT and catecholamine neuronal patterns are nonoverlapping and readily resolved in the larval ventral ganglion (Budnik and White, 1988; Vallés and White, 1988).

The salient feature of the 5HT neuronal pattern in the larval CNS relevant to this work is that, in the ventral ganglion, each hemisegment contains a SHT-IR neuronal cluster; for each cluster, the somata are close, and their axons fasciculate and cross over to the contralateral hemisegment through a transverse commissure and form extensive arbors in the contralateral neuropil.
Genetically mosaic larvae were generated by crossing $D d c^{+}$. RingX/Binsn; Dd ${ }^{n 27} / S M 6$ females with cho $s n / Y$; Ddc $c^{27} / S M 6$ males. Among the progeny, $D d c^{+}$. RingX/cho sn; $D d c^{n 27} / D d c^{n 27}$ zygotes can potentially lose the $D d c^{+} \cdot \operatorname{Ring} X$ chromosome, resulting in a $D d c$ genetic mosaic composed of diplo- $X$ cells that are $D d c^{+}$and haplo- $X$ cells that are $D d c^{-}$; these $D d c$ mosaics were recognized by scoring for phenotypic mosaicism in the larval epidermis as described in the Materials and Methods.

\section{HT immunoreactivity in CNSs of genetically mosaic larvae}

A total of 101 useful mosaic larvae were obtained from 4420 larvae screened. Of these, the majority were third instar (94), and a few were second instar (7). Their CNSs were dissected and immunoreacted with $5 \mathrm{HT}$ monoclonal antibody. In the analyses presented below, immunofluorescence patterns were assessed for the presence or absence of 5HT-IR neuronal cell bodies, axonal projections, and dendritic arbors; special attention was paid to the neurons of the ventral ganglion clusters, because these constitute the most reliable feature, as described above.

One might consider 5HT synthesis in 5HT neurons as one of the phenotypes associated with $D d c^{+}$genotype. However, the presence of 5HT in a neuron may not necessarily be due to endogenous synthesis, because 5HT neurons can also take up $5 \mathrm{HT}$. In other words, $5 \mathrm{HT}$ presence could be either due to $D d c^{+}$ genotype or due to accumulation of exogenously synthesized 5HT. Several possibilities may be expected in a genetically mosaic nervous system composed of $D d c^{+}$and $D d c^{-}$cells.

(1) Secreted 5HT is only available for reuptake to the cell in which it is synthesized. In this situation, the phenotype " $5 \mathrm{HT}$ presence" is cell autonomous, because only $D d c^{+}$neurons will be 5HT-IR. Therefore, because the mosaic boundary in gynandromorphs has a random orientation (reviewed in Hall et al., 1976), CNSs with all types of mosaic patterns (5HT-IR and 5HT-minus) would be observed.

(2) 5 HT secreted by $D d c^{+}$cells is freely available to be accumulated by any $D d c^{-}$cell. Under these circumstances the phenotype would be nonautonomous, resulting in an all-or-none situation: 5HT-neurons will be either all 5HT-IR or all 5HTminus. The latter case represents CNSs in which all $5 \mathrm{HT}$ neurons were $D d c^{-}$.

(3) 5HT secreted from a given cluster of $D d c^{+}$neurons may be only available to some $D d c$ 5HT neurons. In this case, phenotypically mosaic CNSs, that is, consisting of neurons that are 5HT-IR and non-IR, will be observed. In these phenotypic mosaic CNSs, at least some cells that are genotypically $D d c^{-}$ will exhibit 5HT immunoreactivity. This situation would represent a limited degree of autonomy for the " $5 \mathrm{HT}$ presence" phenotype associated with $D d c^{+}$genotype.

Among the $101 \mathrm{CNSs}$ from mosaic larvae, 3 different patterns of 5HT-IR neurons were observed: the wild-type 5HT pattern where all the neuronal clusters are IR (20/101; Fig. 1A), a mutant $D d c^{-}$pattern where the CNS was totally devoid of $5 \mathrm{HT}$ immunoreactivity $(5 / 101$; Fig. $1 B)$, and a mosaic pattern composed of 5HT-IR regions adjacent to regions devoid of 5HT immunoreactivity $(76 / 101$; Fig. $2 A-D)$. The pattern shown in Figure $1 A$, where all neurons are 5HT-IR, could be due to the CNS being genotypically wild type $\left(D d c^{+}\right)$. Alternatively, it could result from either free or limited accessibility of 5HT synthesized in $D d c^{+}$neurons in a genetically mosaic CNS. In the second case (Fig. $1 B$ ), where the CNS is devoid of 5HT immunoreactivity, the CNS must be $D d c^{-}$; thus the mosaic boundary did not go 

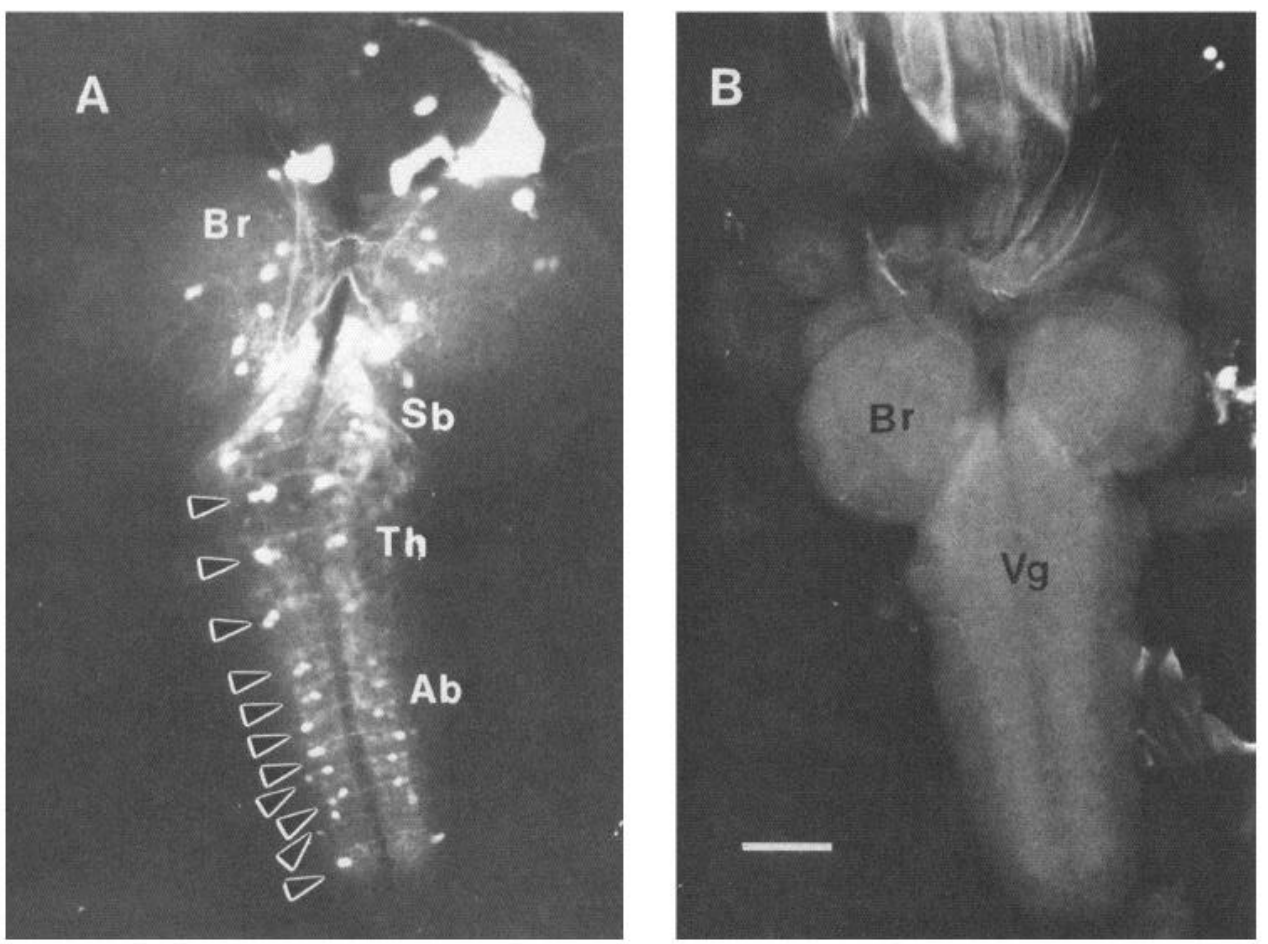

Figure 1. Serotonin immunoreactivity patterns in CNS whole-mounts of mosaic larvae. $A$, This sample represents a CNS showing wild-type pattern of 5HT immunoreactivity. It is photographed to show most of the 5HT neuronal clusters. The Drosophila larval CNS is composed of paired brain lobes $(\mathrm{Br})$ and a compound ventral ganglion $(\mathrm{Vg})$. In the ventral ganglion, a total of 14 clusters are observed: 3 in the subesophageal $(S b)$ region (which are not very well resolved in this photograph), 3 in the thoracic segments $(T h)$, and 8 in the abdominal (Ab) segments. Arrowheads point to the 3 thoracic and the 8 abdominal clusters. The majority of clusters are composed of 2 somata, but the last abdominal cluster is composed of only 1 neuron, while 3 clusters, 2 in the subesophageal region and the prothoracic cluster, are composed of 3 neurons each. There are 4 clusters in the brain lobe. For a detailed description, see Vallés and White (1988). B, This whole-mount, characterized by the complete absence of 5 HT immunoreactivity, represents a $D d c^{-}$pattern. Scale bar, $100 \mu \mathrm{m}$ for $A ; 60 \mu \mathrm{m}$ for $B$.

through the CNS in the individuals of this class. Furthermore, because some CNSs from mosaic larvae, as judged by their cuticular phenotype, were entirely devoid of 5HT immunoreactivity, either 5HT is not synthesized outside of the CNS, or 5HT synthesized in some other tissue is not available to the CNS neurons. These findings are consistent with the findings of Beall and Hirsh (1987) on CNSs carrying genetically engineered variants of the $D d c$ locus with altered neuronal patterns of expression.

In the third group, the phenotypic mosaic boundary traversed through the CNS, resulting in 5HT-IR and 5HT-minus regions. Representative whole-mount samples of mosaic CNSs composed of 5HT-IR and 5HT-minus regions are depicted in Figure $2, A-D$. From the analyses of $5 \mathrm{HT}$ immunoreactivity patterns of the 76 phenotypic 5HT-IR mosaic CNSs, the following conclusions were reached.

(1) 5HT-minus patches of all sizes and varying distribution were observed. Figure $2 A$ shows a mosaic CNS in which only 1 of 4 brain clusters is 5HT-IR, and all clusters in the ventral ganglion are $5 \mathrm{HT}$-IR. Figure $2 B$ shows a mosaic CNS with $5 \mathrm{HT}$ IR brain lobes and a $5 \mathrm{HT}$-minus ventral ganglion. Figure $2 \mathrm{C}$ shows a mosaic CNS in which the posterior ventral ganglion is 5HT-IR (abdominal 5HT-IR neuromeres), but the anterior ventral ganglion and the brain lobes are 5HT-minus. Figure $2 D$ shows a mosaic ventral ganglion with 2 noncontiguous 5HTminus patches in the ventral ganglion.

(2) The mosaic boundary between the 5HT-IR and 5HTminus patches never fell along the longitudinal axis; the left and right hemisegments were always either 5HT-IR or 5HT-minus. This aspect was scored most reliably in the ventral ganglion (Fig. 2A,C,D).

(3) In some hemisegments, the cell bodies had normal levels of 5HT immunoreactivity, but the neuropil showed decreased levels of 5HT immunoreactivity as compared to the contralateral side (discussed below; Figs. $3 A, 4 A$ ).

(4) In some samples, a few neurons with lower than normal levels of 5HT immunoreactivity were observed. Usually, these neurons were at the margins between the 5HT-IR and 5HTminus patches (discussed below; see Fig. $3 A, B$ ).

A logical interpretation of these patterns is that normal 5HT immunoreactivity in the soma, main axonal projections, and dendritic arbor could represent a wild-type $D d c^{+}$cell. Conversely, absence of 5HT immunoreactivity in the expected position of a $5 \mathrm{HT}$ cluster (no IR cell body, axonal projection, or dendritic arbor) could represent a $D d c^{-}$cell to which $5 \mathrm{HT}$ from a $D d c^{+}$ cell is not available. Additionally, some 5HT-IR cells may be genotypically $D d c^{-}$, but $5 \mathrm{HT}$ is available to them; the extent of 5HT immunoreactivity in this case may depend on the amount of $5 \mathrm{HT}$ available, which might correlate with the proximity of $D d c^{+}$cells.

\section{DDC and 5HT immunoreactivity in CNSs of genetically mosaic larvae}

To test the interpretations stated above, we simultaneously immunostained mosaic CNSs with 5HT antibodies and antibodies raised against the Drosophila DDC. Previous immunostaining 

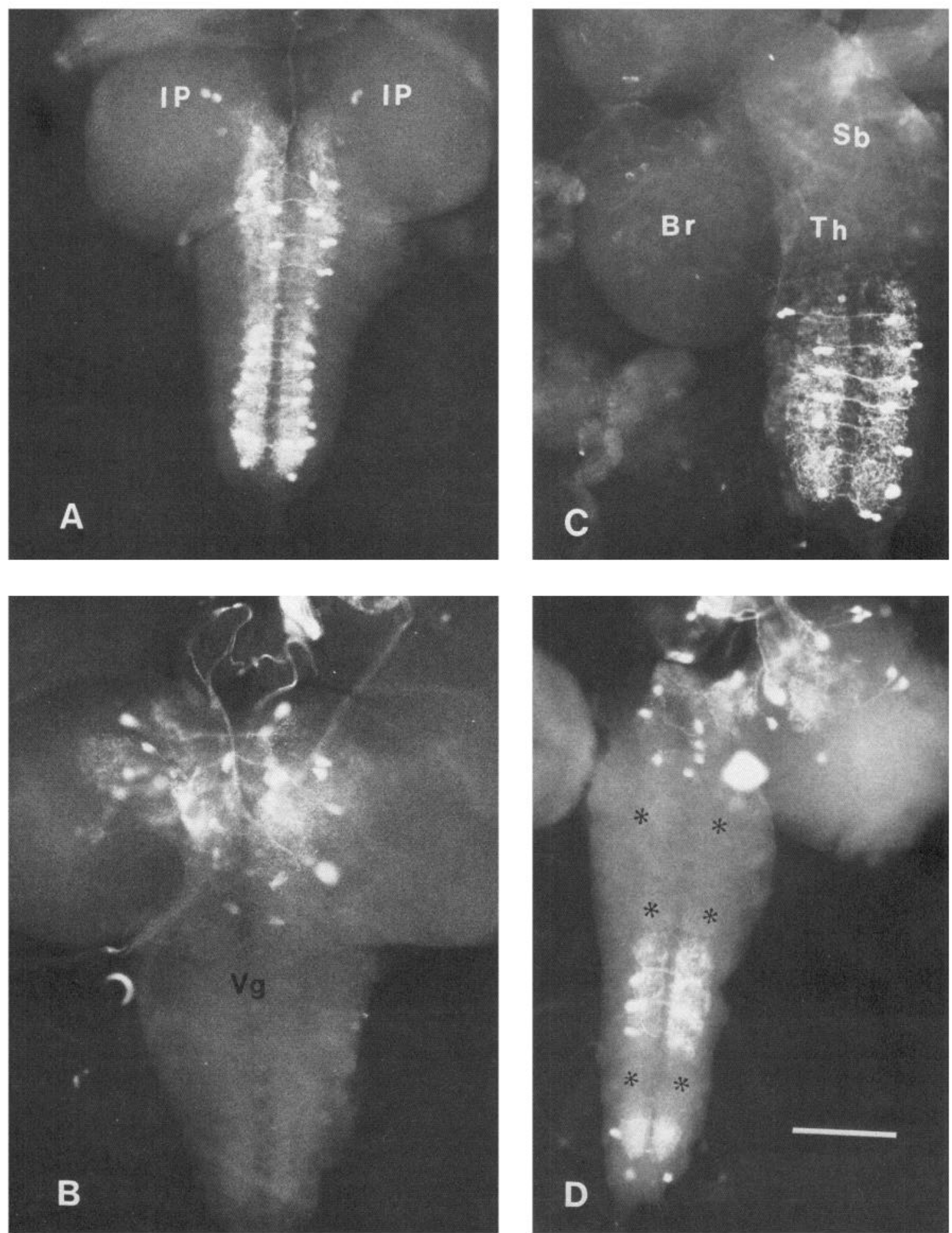

Figure 2. Larval CNSs showing mosaic 5HT immunoreactivity patterns. $A$, Photomicrograph of a mosaic with only $15 \mathrm{HT}$-IR cluster (IP) in the brain lobe; the other 3 clusters are non-IR. Note that in the ventral ganglion all clusters are 5HT-IR. B. Photomicrograph of a mosaic CNS showing $5 \mathrm{HT}$ immunoreactivity in the brain, but a mostly non-IR ventral ganglion $(\mathrm{Vg})$. C. Photomicrograph of a mosaic CNS showing $5 \mathrm{HT}$ immunoreactivity in the abdominal segments only. $B r$, brain lobe; $T h$, thoracic; $S b$, subesophageal. $D$, This mosaic CNS shows an interrupted pattern of 5 HT immunoreactivity. The brain lobes and the subesophageal region are 5HT-IR, but anterior thoracic segments and midabdominal segments are devoid of 5HT immunoreactivity. Asterisks highlight the regions devoid of immunoreactivity. Scale bar, $100 \mu \mathrm{m}$ for $A, C, D ; 80 \mu \mathrm{m}$ for $B$.

studies had shown that mutant $D d c^{-}$genotypes used in this analysis were devoid of DDC immunoreactivity (Beall and Hirsh, 1987). Because the 5HT and catecholamine neuronal patterns are well defined and in the ventral ganglion are distinct, DDC immunoreactivity allowed the identification of those 5HT-IR neurons in the mosaic CNSs that were also $D d c^{+}$. The results of these analyses are described below.

CNSs from 37 mosaic larvae were immunolabeled simulta- 

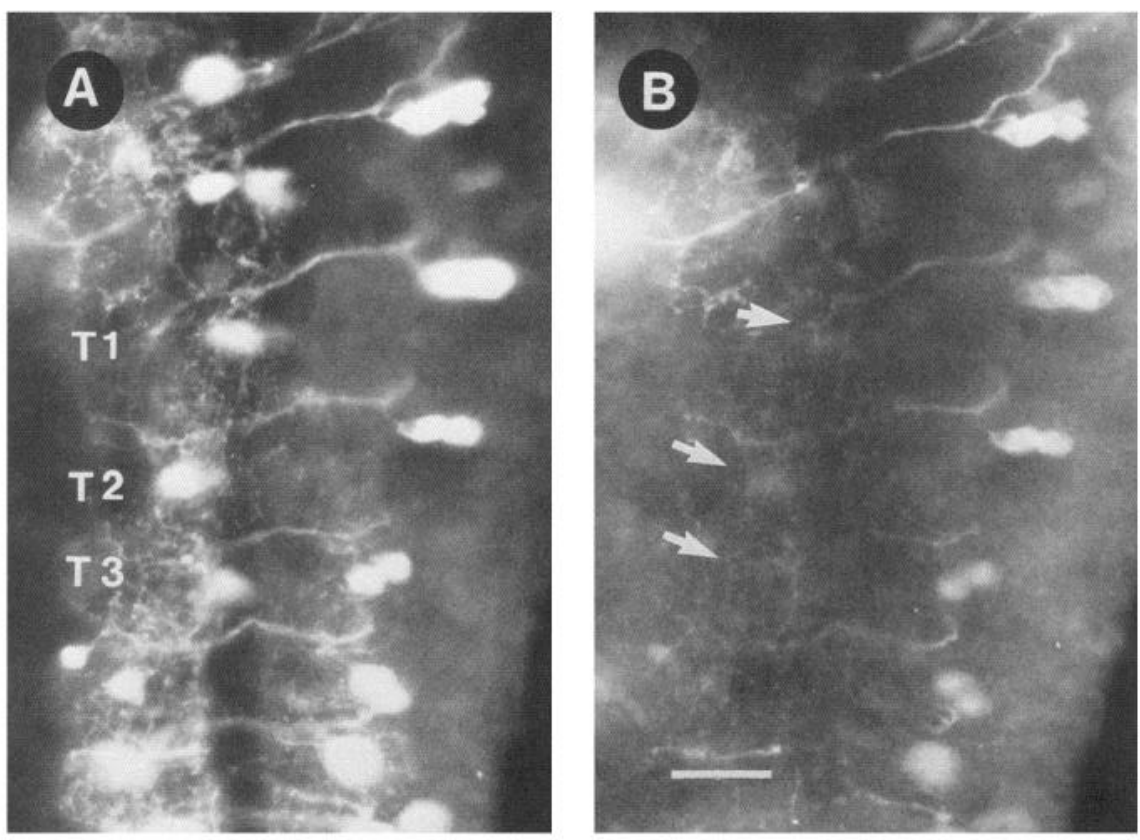

Figure 3. Ddc larval mosaic CNS simultaneously stained with 5HT and DDC antibodies: mosaic whole-mount photographed to show 5HT immunoreactivity $(A)$ and corresponding DDC immunoreactivity $(B)$. These photomicrographs show 6 segments in the ventral ganglion; the plane of focus is on the cell bodies. 5HT-IR clusters are observed in both the left and the right hemisegments, whereas DDC-IR clusters are present only in the right hemisegment. Arrows in $B$ indicate absence of DDC immunoreactivity in $T 1, T 2$, and $T 3$ clusters. Note the high neuropil $5 \mathrm{HT}$ immunoreactivity in the left hemisegments compared to the right hemisegments. Scale bar, $50 \mu \mathrm{m}$.

neously with 5HT and DDC antibodies as described in Materials and Methods. FITC- and rhodamine-conjugated secondary antibodies were used to visualize the 5HT and DDC immunoreactivity patterns, respectively. Two representative samples of mosaic CNSs, showing 5HT-IR and DDC-IR patterns, are pre- sented in Figures 3 and 4. In these preparations, FITC fluorescence indicative of $5 \mathrm{HT}$ immunoreactivity was seen in cell bodies, axons, and neuropil (Figs. $3 A, 4 A$ ), whereas rhodamine fluorescence indicative of DDC immunoreactivity was seen primarily in cell bodies and axons (Figs. $3 B, 4 B$ ). From the ex-
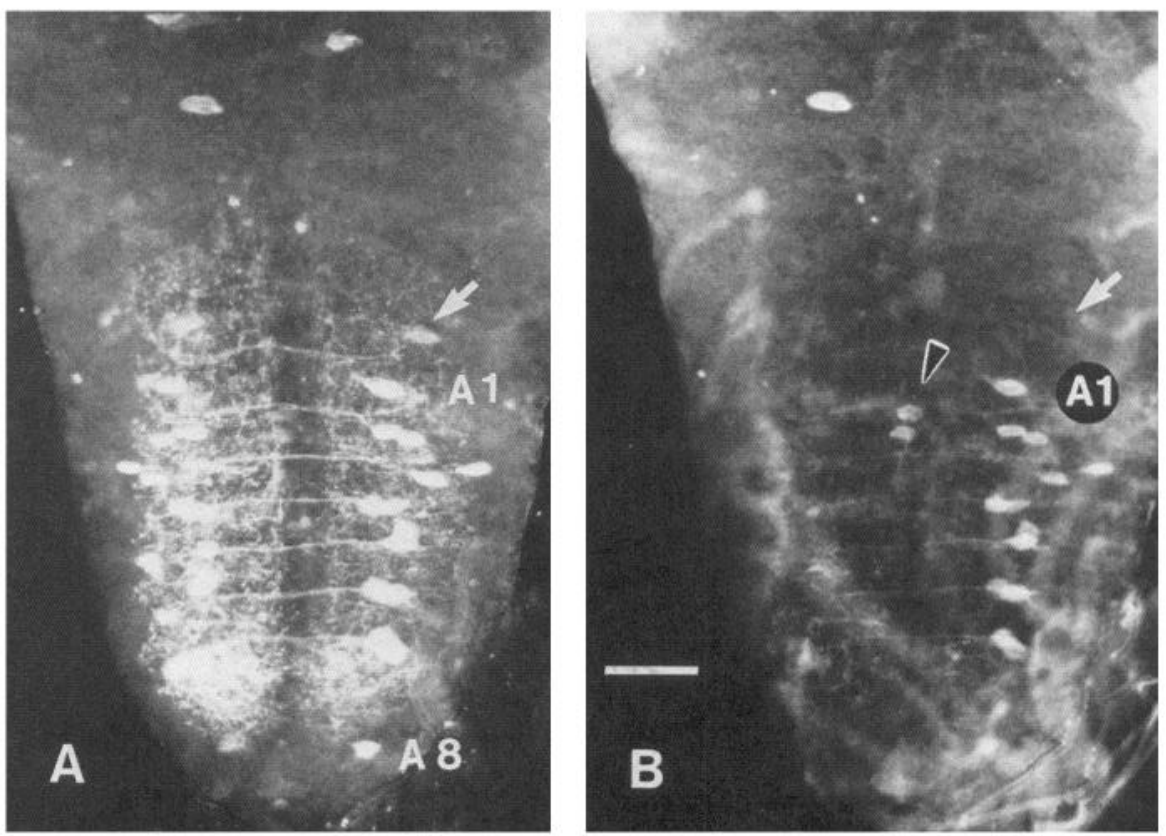

Figure 4. A mosaic whole-mount photographed to show 5HT immunoreactivity $(A)$ and corresponding DDC immunoreactivity $(B)$. These photomicrographs show the abdominal segments $(A 1-A 8)$. Note the absence of DDC immunoreactivity in the left hemisegments and presence of $5 \mathrm{HT}$ immunoreactivity in both left and right neuronal clusters. The intense neuropil immunoreactivity is only in the left hemisegments, contralateral to the right DDC-IR neurons. Neuropil SHT immunoreactivity on the right, contralateral to DDC-minus neurons, is weak. The arrows point to a weakly 5HT-IR neuronal cluster in the right third thoracic segment $(A)$ that is devoid of DDC immunoreactivity $(B)$. Arrowhead in $B$ points to catecholamine-containing DDC-IR neurons. Scale bar, $70 \mu \mathrm{m}$. 
amination and comparison of these patterns, the following conclusions were reached.

(1) 5HT neurons that stained with DDC antibody were always 5HT-IR (Figs. 3, 4).

(2) In contrast to the $5 \mathrm{HT}$ immunoreactivity, where differences in immunoreactivity within the left and right hemisegment were never observed, after staining with the DDC antibody, in the majority of mosaics, a longitudinal boundary traversing between left and right hemisegments was obscrved (31/37). Thus, in these mosaics, DDC-minus neurons were 5HTIR. Figures $3 B$ and $4 B$ depict 2 such mosaics, in which the DDC-IR and DDC-minus boundary is along the longitudinal midline in the ventral ganglion.

(3) In a left-right mosaic for DDC immunoreactivity, very often the neuropil contralateral to the DDC-IR cluster was 5HTIR to a greater extent, compared to the neuropil on the same side, where the $D d c^{-}$neurons arborize. Examples of such mosaics are shown in Figures $3 A$ and $4 A$; the left neuropil in which the right DDC-IR neurons arborize is highly 5HT-IR, while the right neuropil in which the DDC-minus neurons from the left arborize shows lower levels of 5HT immunoreactivity.

(4) 5HT-IR neurons with low levels of immunoreactivity observed at the margins between 5HT-IR and 5HT-minus patches were always DDC-minus (Fig. $3 A, B$ ).

Thcsc data imply that $5 \mathrm{HT}$ synthesized in $D d c^{+}$neurons is not freely diffusible throughout the nervous system, nor is it strictly restricted in an autonomous fashion to only $D d c^{+}$neurons. The finding that, with respect to 5HT distribution, left and right hemisegments were always either 5HT-IR or 5HTminus can be readily explained, if $5 \mathrm{HT}$ synthesized within a given segment is accessible to all 5HT neurons within that segment irrespective of their ability to synthesize $5 \mathrm{HT}$.

\section{Accumulation of $5 H T$ in $\mathrm{Ddc}^{-}$neurons}

The mechanism operating in the genetic $D d c$ mosaic that allows accumulation of 5HT in $D d c^{-}$neurons is not known. The observed pattern suggests that the $D d c^{-}$neurons acquire $5 \mathrm{HT}$ by uptake processes, possibly in a fashion similar to the accumulation seen in $D d c^{-}$CNSs after they have been incubated in exogenous 5HT (Vallés and White, 1986a). Furthermore, the $D d c^{-}$neurons in a mosaic CNS reveal this normally occurring process at physiological concentrations of $5 \mathrm{HT}$ in an in vivo situation. From their morphology, the ventral-ganglion 5HT neurons in the thoracic and abdominal segments appear to be primarily interneurons that arborize most extensively within the contralateral neuropil of their own segment (Vallés and White, 1988). The axons from the 2 paired clusters appear to be in apposition (Vallés and White, 1988). Presumably, some synaptic terminals from $5 \mathrm{HT}$ neurons within a segment may be in close proximity, allowing reuptake of released 5HT by all neurons within that segment. The uptake seen in mosaics probably reveals that normally all neurons within a segment may participate in the removal of synaptically released 5HT. If this is indeed a reflection of normal uptake process, perhaps there is some adaptive advantage to have all 5HT neurons participate in the process.

A limited degree of accessibility to $5 \mathrm{HT}$ would help explain those neurons that were characterized by low 5HT immunoreactivity. These neurons were always DDC-minus and were located in segments adjacent to DDC-IR segments. The distribution of $5 \mathrm{HT}$-IR fibers and varicosities suggest that, though the 5HT neurons have predominantly intrasegmental arbors, some 5HT-IR longitudinal fibers are also observed, suggesting that at least some 5HT neurons have an intersegmental arbor component (Vallés and White, 1988). Presumably, most synaptic terminals from $D d c^{+}$neurons are in close association with the terminals from 5HT neurons in their own segment, but a few are also in apposition with synaptic terminals from $5 \mathrm{HT}$ neurons in an adjacent segment. This would result in a high level of 5HT accessibility to $D d c^{-}$neurons within the same segment and a markedly reduced level of $5 \mathrm{HT}$ accessibility to 5 HT neurons in the adjacent segments.

In Drosophila. genetic mosaics involving mutations that disrupt behaviors have been used in assessing the anatomical parts of the fly that are associated with specific behaviors (reviewed in Hall, 1978a). A mutant gene disrupting a behavior is likely to cause neurochemical and/or morphological change in a set of neurons that ultimately result in the dysfunction of the neurons responsible for the behavior. Such behavioral foci, defined as sites on the blastoderm surface that, when of mutant genotype, result in a mutant phenotype, may behave either as "domineering" or "submissive" (Hotta and Benzer, 1972; Hall, 1978b; von Schilcher and Hall, 1979). In the case of a domineering focus, either member of the pair, when mutant, will result in a mutant phenotype, whereas in the case of a submissive focus, mutant phenotype will result only when both members of the pair are mutant. The analysis presented in this paper provides an example for a physical basis for a submissive focus, because when only one cluster of the 5HT neuronal pair is genotypically wild type $\left(D d c^{+}\right)$, the contralateral cluster is phenotypically wild type (5HT-IR). Presumably these DDC-minus but 5HT-IR neurons could provide wild-type physiological function.

\section{Conclusion}

In conclusion, the analysis presented in this paper demonstrates that, in $D d c$ genetic mosaic CNSs, phenotypic mosaicism for both 5HT and DDC immunoreactivity is observed. 5HT-IR neurons either are $D d c^{+}$or have their synaptic terminals in apposition to other 5HT-IR neurons that are $D d c^{+}$. The presence of $5 \mathrm{HT}$ immunoreactivity in neurons that are devoid of DDC immunoreactivity implies that 5HT is accumulated by reuptake mechanisms from terminals that are in close proximity.

\section{References}

Beall CJ, Hirsh J (1987) Regulation of the Drosophila dopa decarboxylase gene in neuronal and glial cells. Genes Dev 1:510-520.

Budnik V, White K (1988) Catecholamine-containing neurons in Drosophila melanogaster: distribution and development. J Comp Neurol 268:400-413.

Budnik V, Wu C-F, White K (1989) Altered branching of serotonincontaining neurons in Drosophila mutants unable to synthesize serotonin and dopamine. J Neurosci 9:2866-2877.

Gailey DA, Bordne DL, Vallés AM, Hall JC, White K (1987) Construction of an unstable ring- $X$ chromosome bearing the autosomal gene dopa decarboxylase in Drosophila melanogaster and analysis of Ddc mosaics. Genetics 115:305-311.

Gilbert D, Hirsh J, Wright TRF (1984) Molecular mapping of a gene cluster flanking the Drosophila dopa decarboxylase gene. Genetics 106:679-694.

Hall JC (1978a) Behavioral analysis in Drosophila mosaics. In: Genetic mosaics and cell differentiation (Gehring WJ, ed), pp 259-305. Berlin: Springer.

Hall JC (1978b) Control of male reproductive behavior by the central nervous system of Drosophila: dissection of courtship pathway by genetic mosaics. Genetics 92:437-457.

Hall JC, Gelbart WM, Kankel DR (1976) Mosaic systems. In: Genetics and biology of Drosophila Vol la (Ashburner M, Novitski E, eds), pp 265-314. London: Academic. 
Hotta Y, Benzer S (1972) Mapping of behaviour in Drosophila mosaics. Nature 240:527-535.

Konrad KD, Marsh JL (1987) Developmental expression and spatial distribution of dopa decarboxylase in Drosophila. Dev Biol 122:172185.

Livingstone M, Tempel B (1983) Genetic dissection of monoamine neurotransmitter synthesis in Drosophila. Nature 303:67-70.

Vallés AM, Whitc K (1986a) Development of scrotonin-containing neurons in Drosophila mutants unable to synthesize serotonin. J Neurosci $6: 1482-1491$.

Vallés AM, White K (1986b) Serotonin mosaics in larval CNS of Drosophila. Soc Neurosci Abstr 12:1164.

Vallés AM, White K (1988) Serotonin-containing neurons in Dro- sophila melanogaster: development and distribution. J Comp Neurol 268:414-428.

von Schilcher F, Hall J (1979) Neural topography of courtship song in sex mosaics of Drosophila melanogaster. J Comp Physiol 129:8595.

Wright TRF (1987) The genetics of biogenic amine metabolism, sclerotization, and melanization in Drosophila melanogaster. Adv Genet 24:127-222.

Wright TRF, Hodgetts RB, Sherald AF (1976) The genetics of dopa decarboxylase in Drosophila melanogaster. I. Isolation and characterization of deficiencies that delete the dopa-decarboxylase-dosagesensitive region and the $\alpha$-methyl-dopa-hypersensitive locus. Genetics 84:267-285. 